\title{
NGOs - die besseren Unternehmen oder business as usual?
}

\author{
Jutta Joachim $\mathbb{D} \cdot$ Andrea Schneiker
}

Angenommen: 20. Mai 2021 / Online publiziert: 29. Juni 2021

(C) Der/die Autor(en) 2021

Zusammenfassung Nichtregierungsorganisationen (NGOs), so der Befund aus Wissenschaft und Praxis, übernehmen immer häufiger Praktiken und Strukturen von Wirtschaftsunternehmen. In diesem Kontext ist oftmals von einer „Kommerzialisierung“"von NGOs die Rede, die in unterschiedlichen politikwissenschaftlichen Disziplinen erforscht wird. Die Dritte-Sektor-Forschung, die Protest- und Bewegungsforschung, die Forschung zu NGOs in den Internationalen Beziehungen befassen sich jedoch jeweils nur mit Teildimensionen des Kommerzialisierungs-Phänomens und betrachten entweder nur damit verbundene organisationsinterne Veränderungen oder nach außen gerichtetes Organisationshandeln. Daher fehlt es einerseits an Wissen darüber, wie umfassend die Kommerzialisierung von NGOs vorangeschritten ist und welche Wechselbeziehungen es zwischen einzelnen Dimensionen der Kommerzialisierung gibt. Um diese Lücke zu schließen, trägt der Aufsatz nicht nur die unterschiedlichen Erkenntnisse der verschiedenen Disziplinen zusammen, sondern entwickelt auch ein analytisch-konzeptionelles Instrumentarium, auf dessen Basis die diversen Dimensionen von Kommerzialisierung identifiziert und analytisch getrennt voneinander betrachtet werden und Forschungsdesiderata benannt werden können.

Schlüsselwörter NGOs · Unternehmen · Kommerzialisierung · Wettbewerb · Kommodifizierung

Jutta Joachim

Nijmegen School of Management, Dept. of Political Science, Radboud University, 6500 HC

Nijmegen, Niederlande

E-Mail: j.joachim@fm.ru.nl

Andrea Schneiker $(\varangle)$

Zeppelin Universität, Friedrichshafen, Deutschland

E-Mail: andrea.schneiker@zu.de 


\title{
NGOs—better than business companies or business as usual?
}

\begin{abstract}
Academics and practitioners alike have suggested that non-governmental organizations (NGOs) show signs of commercialization as they increasingly adopt practices and exhibit structures of business corporations. While scholars of various sub-disciplines of political science have paid attention to this phenomenon, including third sector research, sociology and International Relations, they have either placed exclusive focus on internal organizational changes or on the external behavior of NGOs. Because of this partial treatment, we thus far lack knowledge not only with respect to the scope of commercialization of NGOs, but also its different facets and the interdependencies that exist between them. This article provides a systematic review of the state of the art and based upon it, develops a conceptual framework of commercialization intended to stimulate future research on the subject.
\end{abstract}

\section{Einleitung}

In der politikwissenschaftlichen Forschung zu nicht-profitorientierten Nichtregierungsorganisationen (NGOs) ist seit einigen Jahren von der „Kommerzialisierung“ dieser Organisationen die Rede. Damit ist gemeint, dass sich gemeinwohlorientierte Organisationen, deren Handeln über ,Menschen, eine Sache oder ein Thema jenseits ihrer eigenen sozialen Bezugsgrenzen“ hinausgeht (Frantz 2010, S. 191; Heins 2002), in ihren Strukturen, Prozessen und Zielen profitorientierten Unternehmen annähern und ähnlich wie diese handeln. Beispielsweise werden humanitäre NGOs nicht zuletzt deshalb von einigen Autor*innen als „Firmen“ (Barnett und Weiss 2008a, S. 17) bezeichnet. Auch von Praktiker*innen wird solch ein Wandel konstatiert. So schreiben die Autoren eines Bandes zum ,strategische[n] Management humanitärer NGOs“, zu denen auch der Geschäftsführer der NGO Islamic Relief Deutschland gehört, in ihrem Vorwort: „Der Sektor der Solidarität und Unterstützung der Notleidenden und Bedürftigen entwickelt und erfordert zusätzlich zu seinen traditionellen Attributen - uneigennütziges Engagement, Handeln aus Überzeugung - auch solche Attribute, die man vorwiegend von kommerziellen Sektoren kennt: Effektivität in den strategischen Zielsetzungen, Effizienz in den Operationen, Transparenz gegenüber den Stakeholdern, Professionalität, Kunden- und Serviceorientierung." (Heuser und Abdelalem 2018, S. v). Doch dieser Trend erstreckt sich auch auf andere Politikfelder, gleichwohl er vermutlich nicht alle Organisationen gleichermaßen erfasst.

Das Phänomen der Kommerzialisierung wird je nach politikwissenschaftlichem Forschungsstrang, in dem NGOs systematisch als eigener Untersuchungsgegenstand im Vordergrund stehen, auf unterschiedliche Weise untersucht. So betrachten die Non-Profit- bzw. die Dritte-Sektor-Forschung, die Forschung zum Ehrenamt und die Protest- und Bewegungsforschung sowie die Forschung zu Internationalen Beziehungen jeweils nicht nur unterschiedliche Dimensionen von Kommerzialisierung, sondern auch unterschiedliche Akteure. Am stärksten ausgeprägt ist die Debatte bislang in der Forschung zum Non-Profit-Sektor. Diese legt den Fokus auf den Wandel der Organisationsstrukturen und -prozesse innerhalb von Organisationen, die, so die 
These, sich jenen von Unternehmen angleichen (z. B. Alexander und Weiner 1998; du Gay et al. 1996, S. 267; Hwang und Powell 2009; Maier und Meyer 2011, S. 738). Dabei liegt das Hauptaugenmerk auf gemeinnützigen Organisationen, die v. a. im Rahmen des Wohlfahrtsstaates Dienstleistungen auf nationaler Ebene erbringen (Hansmann 1980) ${ }^{1}$, jedoch - anders als in den anderen genannten Disziplinen gilt das Interesse der Non-Profit-Forschung nicht nur NGOs, die im Rahmen dieses Aufsatzes die relevante Bezugsgröße sind, sondern beispielsweise auch wohlfahrtsstaatlichen Verbänden und Gewerkschaften (z. B. Thomas 2013) oder auch Einrichtungen wie Krankenhäusern (z.B. Horwitz 1988). Die Forschung zum Ehrenamt untersucht, wie sich die Kommerzialisierung von NGOs auf die Beziehungen zu ihren individuellen Unterstützer*innen auswirkt (z. B. Eberwein und Saurugger 2013) und konstatiert einerseits einen Motivationswandel Letzterer und andererseits ein gewandeltes Angebot für Engagement von Seiten der NGOs (Beher et al. 2001; More-Hollerweger 2014; Priller 2011, S. 16; Rahmann 1999). Dieser Befund deckt sich mit dem der Protest- und Bewegungsforschung, auch wenn diese weniger organisational verfasste Akteure und vielmehr lose und zeitlich begrenzte Netzwerke betrachtet. Die NGO-Forschung in der Teildisziplin der Internationalen Beziehungen (IB) schließlich befasst sich zwar auch mit nicht-profitorientierten Organisationen, aber vorwiegend mit transnational handelnden NGOs, deren Beneficiaries - anders als im Falle von nationalstaatlich verhafteten Non-Profit-Organisationen - in anderen lokalen Kontexten verortet sind als die Organisationen selbst, ihr Personal und ihre Unterstützer*innen. Diese Forschung ist vor allem daran interessiert, wie sich Kommerzialisierung in den Strukturen, im Rahmen derer NGOs handeln, äußert und nimmt daher die Beziehungen zwischen NGOs untereinander und zu anderen Akteuren sowie die sich daraus ergebenden Folgen für das Handeln der Organisationen in den Fokus. Eine zentrale Annahme hierbei ist, dass die NGOs untereinander im Wettbewerb um Fördergelder stehen und dies zu suboptimalen und dysfunktionalen Ergebnissen führt (z.B. Cooley und Ron 2002). Organisationsinterne Prozesse und Strukturen werden in den IB jedoch meist vernachlässigt. Daher ist eine Berücksichtigung des Forschungsstandes in anderen Teilen der Politikwissenschaft insbesondere für die IB-Perspektive auf NGOs gewinnbringend. Obwohl sich alle diese Forschungsstränge mit den unterschiedlichen Ausprägungen des gleichen Phänomens befassen, nehmen sie wenig Bezug aufeinander und finden entsprechende Debatten hierzu isoliert voneinander statt. Daraus ergeben sich zwei zentrale Schwachstellen in der Literatur.

Erstens erschwert die fragmentierte Betrachtung, die gesamte Reichweite der Kommerzialisierung von NGOs zu erfassen (Maier et al. 2016, S. 64) und beispielsweise zu bestimmen, ob sich von einer grundsätzlichen Wesensveränderung der NGOs sprechen lässt. Die IB-Forschung zu NGOs geht zum Beispiel davon aus, dass Kommerzialisierung Folgen für das Handeln der NGOs hat, blendet aber mögliche Auswirkungen auf die Organisationen selbst aus. Auch erschwert die Betrachtung einzelner - in der Regel jedoch parallel ablaufender - Prozesse der Kommerzialisierung, Aussagen über Kausalzusammenhänge zu treffen. So bleibt unter

\footnotetext{
${ }^{1}$ Es gibt jedoch sehr unterschiedliche Konzeptionalisierung des Dritten Sektors, s. Salmon und Sokolowski (2016).
} 
anderem unterbelichtet, ob organisationsinterne Veränderungen eine Reaktion auf sich wandelnde äußere Rahmenbedingungen sind oder ob sich interne und externe Dimension vielmehr gegenseitig bedingen. Auch fehlt es an Wissen darüber, inwiefern sich Spender*innen an sich verändernde NGOs anpassen oder aber auch selbst mit ihrem Verhalten Anpassungsdruck erzeugen und inwiefern die konstatierte Kommerzialisierung eine eigene Dynamik entfaltet. Zudem ist unklar, welche Konsequenzen sich aus der Kommerzialisierung für die wissenschaftliche Auseinandersetzung mit NGOs ergeben und ob NGOs mit einem veränderten theoretischen und methodischen Instrumentarium als bisher untersucht werden müssen.

Zweitens führt die nach Disziplinen getrennte Betrachtung jeweils einzelner Aspekte von Kommerzialisierung zu einem Nebeneinander zahlreicher Begriffe zur Bezeichnung des Phänomens. So finden neben dem Begriff „Kommerzialisierung“ auch der Terminus „Professionalisierung“ und die Umschreibung ,Firmen ähnliches Verhalten“ sowie - vor allem im angelsächsischen Raum - die Begriffe ,marketization“ und „corporization“ Anwendung (siehe hierzu Maier et al. 2016; Joachim und Schneiker 2018b). Diese Begriffe werden jedoch nicht systematisch zur Erfassung der unterschiedlichen Dimensionen von Kommerzialisierung genutzt, nicht einheitlich definiert und teilweise synonym verwandt. Wir erachten jedoch den Begriff der Kommerzialisierung als Oberbegriff am geeignetsten, weil er es erlaubt unterschiedliche Dimensionen - Strukturen, Handeln, Prozesse, Ziele und Inhalte - zu subsummieren, ohne eine Präferenz für eine bestimmte Dimension zu implizieren.

Diese Blindstellen sind Anlass für den hier vorliegenden Aufsatz, der auf der einen Seite das Anliegen hat, die verschiedenen Forschungsstränge so zusammenzuführen, dass auf konzeptionell-begrifflicher Ebene unterschiedliche Dimensionen von Kommerzialisierung identifiziert und analytisch getrennt voneinander betrachtet werden können. Darauf aufbauend kann dann in zukünftiger Forschung systematisch erfasst werden, in welchem Maße Kommerzialisierung in der Praxis fortgeschritten ist und inwiefern es Unterschiede zwischen einzelnen NGOs und/oder Politikfeldern gibt. Auf der anderen Seite soll durch diese Differenzierung das Phänomen der Kommerzialisierung in seiner Breite dargelegt werden, um deutlich zu machen, welche Zusammenhänge, mögliche Wechselwirkungen und Forschungsdesiderata sich daraus ergeben.

Im Folgen entwickeln wir daher auf Basis der unterschiedlichen Forschungsstränge zunächst eine Typologie unterschiedlicher Dimensionen von Kommerzialisierung und illustrieren diese anhand empirischer Beispiele aus diversen Politikfeldern. Danach zeigen wir auf, welche Differenzen sich aus der unterschiedlichen Betrachtung auf Kommerzialisierung mit Blick auf Triebfedern und Folgen dieser Entwicklung ergeben und welche Fragen sich für die Forschung stellen.

\section{Unterschiedliche Dimensionen von Kommerzialisierung}

Im Folgenden verwenden wir den Begriff der Kommerzialisierung als übergeordnetes Konzept, das sich in verschiedene Dimensionen ausdifferenzieren lässt, die mit jeweils unterschiedlichen Begriffen erfasst werden können (Tab. 1) (Joachim und Schneiker 2018b). Die Dimensionen haben wir deduktiv aus unterschiedlichen 
Tab. 1 Unterschiedliche Dimensionen von Kommerzialisierung

\begin{tabular}{|c|c|c|c|}
\hline Dimension & Beschreibung & Indikatoren & $\begin{array}{l}\text { Vorwiegendes For- } \\
\text { schungsgebiet }\end{array}$ \\
\hline $\begin{array}{l}\text { Korporatisierung } \\
\text { der Organisati- } \\
\text { onsstrukturen }\end{array}$ & $\begin{array}{l}\text { Dezentralisierung } \\
\text { und Ausdifferen- } \\
\text { zierung }\end{array}$ & $\begin{array}{l}\text { Etablierung von Organisations- } \\
\text { einheiten, die auf Einwerbung } \\
\text { und Verwaltung von Geldern } \\
\text { spezialisiert sind }\end{array}$ & $\begin{array}{l}\text { Non-Profit Organisa- } \\
\text { tionen/Dritte-Sektor- } \\
\text { Forschung }\end{array}$ \\
\hline \multirow{2}{*}{$\begin{array}{l}\text { Organisations- } \\
\text { interne } \\
\text { Rationalisie- } \\
\text { rungsprozesse }\end{array}$} & Professionalisierung & $\begin{array}{l}\text { Bezahltes Personal mit fach- } \\
\text { spezifischer (oft akademischer) } \\
\text { Ausbildung }\end{array}$ & $\begin{array}{l}\text { Non-Profit Organisa- } \\
\text { tionen/Dritte-Sektor- } \\
\text { Forschung }\end{array}$ \\
\hline & Standardisierung & $\begin{array}{l}\text { Etablierung vergleichbarer } \\
\text { Praktiken, Regeln, Standards } \\
\text { über NGOs hinweg }\end{array}$ & $\begin{array}{l}\text { Non-Profit Organisa- } \\
\text { tionen/Dritte-Sektor- } \\
\text { Forschung }\end{array}$ \\
\hline \multirow[t]{2}{*}{ Wettbewerb } & $\begin{array}{l}\text { Anzahl an NGOs } \\
\text { Finanzielles } \\
\text { Volumen }\end{array}$ & $\begin{array}{l}\text { Nimmt zu } \\
\text { Nimmt zu }\end{array}$ & \multirow[t]{2}{*}{$\begin{array}{l}\text { NGO-Forschung in den } \\
\text { IB, Non-Profit } \\
\text { Organisationen/Dritte- } \\
\text { Sektor-Forschung }\end{array}$} \\
\hline & $\begin{array}{l}\text { Vergabestrukturen } \\
\text { von Aufträgen } \\
\text { durch Geber }\end{array}$ & Kompetitiv & \\
\hline Kommodifizierung & $\begin{array}{l}\text { NGO wird zum } \\
\text { Produkt }\end{array}$ & $\begin{array}{l}\text { Übernahme von Techniken/ } \\
\text { Praktiken des Marketing und } \\
\text { Branding }\end{array}$ & $\begin{array}{l}\text { Transnationale Bewe- } \\
\text { gungs- und NGO-For- } \\
\text { schung, Non-Profit-Orga- } \\
\text { nisationen/Dritte-Sektor- } \\
\text { Forschung }\end{array}$ \\
\hline \multirow[t]{2}{*}{ Konsumption } & $\begin{array}{l}\text { Beziehungen } \\
\text { zwischen NGO } \\
\text { und Unterstüt- } \\
\text { zer*innen }\end{array}$ & $\begin{array}{l}\text { NGOs appellieren an Eigennutz } \\
\text { der Unterstützer*innen }\end{array}$ & $\begin{array}{l}\text { Non-Profit Organisa- } \\
\text { tionen/Dritte-Sektor- } \\
\text { Forschung, Protest- und } \\
\text { Bewegungsforschung }\end{array}$ \\
\hline & $\begin{array}{l}\text { Beziehungen } \\
\text { zwischen NGOs } \\
\text { und institutionel- } \\
\text { len Gebern }\end{array}$ & $\begin{array}{l}\text { NGOs richten Handeln nicht } \\
\text { nach Bedürftigkeit aus, sondern } \\
\text { nach Interessen der Geber }\end{array}$ & IB-Forschung zu NGOs \\
\hline
\end{tabular}

Literatursträngen abgeleitet. ${ }^{2}$ Wir begreifen sie nicht als exklusiv, sondern als miteinander verknüpft. Der Blick in einzelne Forschungsstränge zeigt dabei nicht nur, dass die konzeptionell-begriffliche Ausdifferenzierung von Kommerzialisierung in einigen Disziplinen weiter vorangeschritten ist als in anderen, sondern auch, dass die einzelnen Begriffe oft nur in der jeweiligen Disziplin verwandt werden, wenngleich sie auch für andere fruchtbar sein können.

\subsection{Korporatisierung}

Mit „Korporatisierung“ wird in der Forschung zu Non-Profit-Organisationen, die sich mit Kommerzialisierung insbesondere mit Blick auf das „Innenleben“ von NGOs befasst, die Übernahme von Organisationsstrukturen bezeichnet, die einem

\footnotetext{
2 Andere Autor*innen, z.B. Maier et al. (2016) kommen zu einer anderen Einteilung. Unser Vorschlag der Einteilung ergibt sich aus der Zusammenführung unterschiedlicher Literaturstränge und berücksichtigt zum Beispiel auch die in der IB-Forschung behandelten Dimensionen von Kommerzialisierung und weicht daher von anderen Einteilungen ab.
} 
unternehmerischen Modell entsprechen (Alexander und Weiner 1998; Horwitz 1988). Korporatisierung manifestiert sich in einer Dezentralisierung und Ausdifferenzierung (Hwang und Powell 2009, S. 270) und damit verbunden der Etablierung von solchen Organisationseinheiten, die sich professionell und oft ausschließlich mit Aufgaben befassen, wie sie bei Unternehmen zu finden sind und die vorranging auf die Einwerbung und Verwaltung von Geldern ausgerichtet sind: Diese Aufgaben umfassen beispielsweise die Kommunikation, Public Relations (PR), Werbung, Marketing, Fundraising, Controlling, Audits und Compliance. Diese Entwicklung wird auch in der IB-Literatur zu NGOs zur Kenntnis genommen, jedoch weitgehend deskriptiv als Teil von Professionalisierung verstanden (Eberwein und Saurugger 2013; Heins 2008, S. 2; Vestergaard 2008). In engem Zusammenhang mit der Korporatisierung, aber doch eine andere Dimension von Kommerzialisierung umfassend, stehen organisationsinterne Rationalisierungsprozesse (Hwang und Powell 2009, S. 268; Strachwitz 2000, S. 27).

\subsection{Organisationsinterne Rationalisierungsprozesse}

Ebenso wie Korporatisierung werden organisationsinterne Rationalisierungsprozesse als Dimension der Kommerzialisierung in der Forschung zu Non-Profit-Organisationen untersucht (Hwang und Powell 2009). Diese Prozesse äußern sich insbesondere in einer Professionalisierung und Standardisierung. Letztere umfasst die Übernahme von extern oder organisationsübergreifend festgelegten vergleichbaren Praktiken, Regeln und Standards. Zur Standardisierung gehören beispielsweise von NGOs häufig gemeinsam entwickelte Verhaltensstandards, wie die „Verhaltensgrundsätze für die Internationale Rot Kreuz und Roter Halbmond Bewegung sowie Nichtregierungsorganisationen (NROs) in der Katastrophenhilfe“ (Diakonie Katastrophenhilfe 1993). Zudem existieren auch sektorübergreifende Standards, zum Beispiel solche, die der Vergabe von Spendensiegeln zugrunde liegen, zum Beispiel dem Spendensiegel DZI. NGOs, die dieses Siegel erhalten haben, sind nach Angaben des Deutschen Zentralinstituts für soziale Fragen (DZI) ,leistungsfähig, arbeiten transparent, wirtschaften sparsam, informieren sachlich und wahrhaftig und haben wirksame Kontroll- und Aufsichtsstrukturen“"(DZI 2020).

Standardisierung und Professionalisierung werden auch in der IB-Literatur zu NGOs diskutiert, allerdings bislang als eher eigenständige und von Kommerzialisierung unabhängige Tendenzen. Standardisierung wird dabei vor allem mit Blick auf die Strukturen, in die NGOs eingebettet sind, behandelt. Insgesamt sind viele Prozesse der Standardisierung auf die Steigerung von Effizienz und Effektivität ausgerichtet (Thaut et al. 2012, S. 148) und ziehen weitere Rationalisierungsprozesse nach sich, wie die Einführung einer sowohl internen als auch externen Evaluation sowie, damit verbunden, einer Rechenschaftspflicht (Gross Stein 2008). So heißt es im Jahresbericht der Welthungerhilfe zum Jahr 2018 beispielsweise, dass externe Evaluationen der Projekte der Organisation u. a. auf Basis der „Kriterien ,Relevanz“, ,Effektivität', ,Effizienz', ,Nachhaltigkeit‘ [...] ,Wirkungen““ (Deutsche Welthungerhilfe e. V. 2019, S. 27) erfolgen. Darüber hinaus können organisationsinterne Rationalisierungsprozesse zu einer Anpassung der Organisationsstrukturen im Sinne einer Korporatisierung führen und umgekehrt. So kann Standardisierung auch die 
Etablierung von organisationsinternen Einheiten zur Messung und Überprüfung eines entsprechenden Organisationshandelns umfassen, zum Beispiel von Abteilungen für Controlling und Compliance.

Mit einer Standardisierung bestimmter Handlungsprozesse, zum Beispiel im Projektmanagement, und der Etablierung von Organisationseinheiten, die mit den dazugehörigen Arbeitsschritten befasst sind, geht häufig eine Professionalisierung der Mitarbeiter*innen der NGOs einher. Gemeint ist damit meist, dass sich NGOs in ihrer Arbeit immer mehr auf bezahltes Personal mit einer fachspezifischen Expertise und einer (oft universitären) Ausbildung stützen (Lang 2013, S. 71). So heißt es zum Beispiel auf der Website des BUND unter der Rubrik „Über uns“: „Kommunikationsprofis, Finanzexpert*innen, Fachfrauen und -männer aller Art und Verwaltungsspezialist*innen sorgen für ökologische Impulse in Politik, Wirtschaft und Gesellschaft.“ (BUND 2020) In diesem Zusammenhang zeigt die folgende Aussage der Geschäftsführerin der NGO Help, wie Professionalisierung, Standardisierung und Korporatisierung zusammenhängen: „Das Finanzmanagement auf Projektebene ist mittlerweile zu einem zeit-, kosten-, und wissensintensiven Bereich des Projektmanagements angewachsen. [...] War Finanzmanagement vor 20 Jahren oftmals nicht mehr als ein einfacher Eintrag in Bücher oder eine Excel-Buchhaltung, wird heute mit komplexen, technisch anspruchsvollen und spezifisches Wissen verlangenden Buchhaltungssystemen gearbeitet“ (Settele 2018, S. 83-84). Auch der stellvertretende Geschäftsführer von Islamic Relief Deutschland erklärt: „Während früher die persönliche Motivation und die Identifikation mit der eigenen Organisation im Vordergrund standen, spielen heute verstärkt die fachlichen Qualifikationen und auch Karriereaspekte beim Personalmanagement eine Rolle“ (Almadhoun 2018, S. 198).

Im Gegenzug stützen sich NGOs immer weniger auf Freiwillige, die bis in die 1980er-Jahre hinein noch den Hauptteil der in NGOs aktiven Personen stellten (Frantz und Martens 2006: 62). Entsprechend wurden Freiwillige als ,,Verbindungsglied" (Glagow 1993, S. 319) zwischen den NGOs und der Gesellschaft verstanden und als ein Unterscheidungsmerkmal von NGOs gegenüber anderen Akteuren. So erklärt zum Beispiel Manfred Glagow in einem Beitrag über entwicklungspolitische NGOs (die in dem folgenden Zitat als NRO abgekürzt werden) aus dem Jahr 1993: „In qualitativer Hinsicht unterscheidet sich das Personal von NRO gegenüber anderen Organisationen dahingehend, daß die z.B. in Marktorganisationen geltenden Professionalisierungsstandards hier nicht unbedingt gültig sind. [...] Es fehlen [...] die technisch-naturwissenschaftlichen Spezialisierungen, die häufig Grundsteine für ein hohes Professionalitätsniveau bilden“ (Glagow 1993, S. 309). In Anbetracht gegenwärtiger Entwicklungen der Professionalisierung scheint die von Glagow unternommene Abgrenzung von NGOs nicht mehr gänzlich angemessen. Denn ähnlich wie in den bereits oben angeführten Beispielen von BUND, Help und Islamic Relief lassen sich auch bei anderen NGOs Hinweise für eine Professionalisierung finden. Dies betrifft nicht nur die Anforderungsprofile für Festangestellte in Stellenanzeigen der Organisationen, sondern auch Anforderungen an Ehrenamtliche, wie Lundström auf Basis einer Studie zu einer Telefonhotline einer Kinderhilfsorganisation in Schweden feststellt. Während Freiwillige für diese Tätigkeit Mitte der 1970er-Jahre keine bestimmte Expertise aufweisen mussten, erwartete die Organisation 20 Jahre später von den Freiwilligen spezialisiertes Wissen über Kinder und über Interviewtechni- 
ken (Lundström 2001, S. 364). Zu diesem Befund aus der Forschung zum NonProfit-Sektor fehlt es bislang an entsprechenden Daten aus der IB-Forschung.

Kommerzialisierung betrifft neben Veränderungen im Inneren von NGOs auch die Beziehungen der NGOs untereinander sowie zu institutionalisierten Gebern, individuellen Spender*innen und Hilfsempfänger*innen.

\subsection{Wettbewerb}

In der IB-Forschung zu NGOs, die sich vor allem mit den Strukturen, innerhalb derer NGOs agieren und die sie zu beeinflussen suchen sowie den Beziehungen von NGOs untereinander und $\mathrm{zu}$ anderen korporativen Akteuren wie Internationalen Regierungsorganisationen (IOs) und Staaten befasst, wird Kommerzialisierung weit weniger differenziert betrachtet als in der Organisationsforschung. In der relevanten IB-Forschung wird Kommerzialisierung relativ undifferenziert gleichgesetzt mit einem zunehmenden Wettbewerb zwischen NGOs um Fördergelder und Projekte. Dieser Wettbewerb setzt sich aus Sicht der IB-Forschung zu NGOs aus drei Elementen zusammen.

Die Anzahl an NGOs ist insbesondere nach Ende des Kalten Krieges rasant angestiegen, von (nach Zählweise der Union of International Associations) weltweit 13.232 Organisationen im Jahr 1981 auf 22.334 im Jahr 1990 und 45.674 im Jahr 2000 (Götz 2011). Obwohl sich dieser Prozess seitdem etwas verlangsamt hat, lässt sich dennoch ein stetiges Wachstum verzeichnen, von 54.977 NGOs im Jahr 2009 auf 61.682 im Jahr 2016 (Joachim 2020, S. 350). Zweitens hat sich auch das finanzielle Volumen zumindest in einigen Bereichen, in denen NGOs aktiv sind, in den letzten Jahren deutlich vergrößert. Ein Beispiel hierfür ist die humanitäre Hilfe. In diesem Bereich ist die offiziell geleistete humanitäre Hilfe zwischen 1990 und 2017 von 2,1 Mrd. USD auf über 20 Mrd. USD angestiegen (ALNAP 2018, S. 83; Collinson und Elhawary 2012, S. 10), wovon ein signifikanter Teil über NGOs abgewickelt wird (Riddell 2008, S. 317-318). Deren Wettbewerb untereinander geht nicht in erster Linie auf den Anstieg des finanziellen Volumens zurück, sondern auf die damit verbundenen gestiegenen Ansprüche der Geber nach einem effektiven und effizienten Einsatz der Gelder (Gourevitch und Lake 2012). Drittens haben, oftmals damit zusammenhängend, insbesondere staatliche Geber teilweise kompetitive Strukturen zur Vergabe von Geldern und Aufträgen etabliert (Pratt et al. 2006; Wen und Chong 2014). Doch nicht nur die Forschung, sondern auch Vertreter*innen aus der Praxis konstatieren einen ,zunehmend härteren Wettbewerb um Aufmerksamkeit und Spendergunst“" (Heuser und Abdelalem 2018, S. v).

\subsection{Kommodifizierung}

Aus Sicht von Forschung zu transnationalen NGOs, Bewegungen und Advocacy ist ein Teil von Kommerzialisierung, dass NGOs sich selbst und ihre Dienstleistungen mittels eines ,,assertive brand asset management“" (van Ham 2008: 131) und entsprechender Techniken aus dem Bereich des Marketing und Branding (Barakso 2010; Vestergaard 2008) als ein Produkt vermarkten, um sich von anderen abzugrenzen und dabei ihre ,unique selling points“ hervorheben. So erläutert der Leiter Marketing 
der deutschen Sektion der NGO Handicap International mit Blick auf die Einführung eines strategischen Marketings 2012: „Die Positionierung bestimmt den Platz im Markt, bei dem das zu bewerbende Konstrukt gegenüber Konkurrenzangeboten hinreichend differenziert wird. Sie spiegelt das unverwechselbare Nutzenangebot wider, die Unique Selling Proposition (USP). Durch den USP soll in erster Linie eine Alleinstellung im Markt durch einen einzigartigen Verkaufsvorteil hervorgehoben werden. Die Positionierung von Handicap International, mit der der Platz der Organisation auf dem Non-Profit-Markt definiert werden soll, lautet in Kürze wie folgt: Handicap International ist der Experte zugunsten von Menschen mit Behinderung weltweit.“ (Schiffelmann 2018, S. 130-131) Eine entsprechende „Profilierung“ der Organisation verlange zudem eine ,aussagekräftige Corporate Identity“, die sich aus den Elementen „Corporate Design“, „Corporate Behaviour“ und „Corporate Communications“ zusammensetzt und auf deren Basis die „Schwerpunkte für die Umsetzung der Werbebotschaft von Handicap International zu bestimmen" sind (Schiffelmann 2018, S. 129-130). Eine auf einer Inhaltsanalyse basierende Studie der Homepages von humanitären NGOs stellt fest, dass diese sich häufig als Organisationen vermarkten, die im Unterschied zu anderen Hilfsakteuren über die meiste Erfahrung verfügen oder am effektivsten in der Bereitstellung von humanitärer Hilfe sind (Joachim und Schneiker 2018b). Ein eigenes Markenzeichen bzw. Label kann, wie der Fall Amnesty International (AI) verdeutlicht, aus bestimmten Symbolen bestehen, wie die von Stacheldraht umgebene Kerze. Zudem kann Branding ebenso routinierte Handlungsweisen umfassen (Barakso 2010), wie die von AI eingeführten „letter writing campaigns“: das Briefeschreiben von Aktivist*innen an Regierungen, um politischen Gefangenen zu helfen. Diese Merkmale sind das Aushängeschild von $\mathrm{Al}$, mit dem sich die Organisation nicht nur weltweit und analog zu einem Produkt in das allgemeine Gedächtnis eingeprägt hat, sondern mit dem sie sich auch von anderen Wettbewerbern unterscheidet und absetzt (Barakso 2010; Dietrich 2018, S. 118). Gleiches gilt im Bereich Umweltschutz für die NGO Greenpeace, für die ,aufsehenerregende Aktionen [...] das Markenzeichen ...“ (Bundeszentrale für politische Bildung o.J.) sind, mit denen sie gleichzeitig ihre Ziele verfolgt und um Unterstützung wirbt.

Darüber hinaus bieten NGOs auch - wie Unternehmen - Waren zum Verkauf an. Wer als Einzelperson die Arbeit von NGOs unterstützen möchte, hat zum Beispiel die Option, Produkte der NGO zu kaufen. Viele NGOs haben dazu auf ihrer jeweiligen Homepage einen eigenen ,Shop“ oder „Store“. Die Tierschutz-NGO PeTA verkauft zum Beispiel T-Shirts, auf denen neben Motiven auch der Schriftzug der Organisation abgedruckt ist (PeTA 2020). Somit machen Unterstützer*innen durch das Tragen des Shirts gleichzeitig Werbung für die NGO. Dabei werden individuelle Unterstützer*innen oftmals als Kund*innen oder Konsument*innen statt als Aktivist*innen angesprochen.

\subsection{Konsumption}

Diese marktähnlichen Beziehungen zwischen NGOs und anderen Akteuren soll hier als Konsumption bezeichnet werden. Das oben genannte Beispiel von PeTA zeigt, dass Konsument*innen nicht nur eine materielle, sondern auch eine ideelle „Ware“ 
angeboten wird. Denn sie können mit dem Kauf, dem Verschenken und dem Tragen eines Produktes einer NGO auch eigennützige Interessen bedienen. Während eine Geldspende für die Organisation in der Regel nach außen nicht sichtbar ist, kann jede/r durch das Tragen eines T-Shirts mit dem Schriftzug einer NGO ihre/seine Unterstützung für diese Organisation und die Werte und Ziele für die sie steht, nach außen kommunizieren. Dies gilt auch für das sharen, liken etc. von Inhalten und Produkten von NGOs auf sozialen Medien. Ein PeTA-T-Shirt wird von der NGO zudem als Produkt für ,,angesagte Kids“ (PeTA 2020) beworben, womit diejenigen, die ein solches T-Shirt erwerben, verschenken und/oder tragen, gleichzeitig eine Aufwertung ihres Selbstwertgefühls oder ihres Status gegenüber ihren gleichaltrigen Peers erfahren können. Somit dient das Engagement, in diesem Falle für den Tierschutz, nicht mehr nur der Sache an sich, sondern bietet Unterstützer*innen die Möglichkeit, eigenen Nutzen aus ihrer Solidarisierung mit den Zielen der NGO zu ziehen.

Die mit der Kommodifizierung verbundene Konsumhaltung von Unterstützer*innen von NGOs wird in der IB-Forschung zu NGOs kaum thematisiert, was nicht überrascht, da diese Forschung den Fokus auf korporative Akteure und kollektives Handeln und weniger auf darin involvierte Individuen legt. In der Forschung zum Ehrenamt wird dies jedoch umfassend beforscht und als Ausdruck des Motivationswandels und als Teil des so genannten ,neuen Ehrenamtes“ verstanden, das sich vom ,alten Ehrenamt“ unterscheidet. Während Letzteres eher von altruistischen und gemeinwohlorientierten Gründen geprägt ist und im Rahmen von etablierten Organisationen stattfindet, wird das neue Ehrenamt vor allem von solchen (oft jungen) Menschen genutzt, die von Motiven wie der Selbstverwirklichung oder einer Problemorientierung geleitet Initiativen in freier Trägerschaft bevorzugen und sich auch eher spontan und kurzfristig für ein Ehrenamt entscheiden (Han-Broich 2012, S. 81; Priller 2011, S. 16; Rahmann 1999). Auch wenn diese Unterscheidung idealtypisch ist und sich die unterschiedlichen Motive und Formen des Engagements nicht unbedingt ausschließen (Beher et al. 2001, S. 262; More-Hollerweger 2014, S. 306), so deckt sich das so genannte neue Ehrenamt doch mit dem in der Protestund Bewegungsforschung konstatierten Wandel politischem Engagements im Zuge der Debatten zur „Postdemokratie“ (Blühdorn 2013) und zum politischen Konsum.

Beruhend auf dieser Forschung ist politisches Engagement nicht mehr (primär) auf Gestaltung und Veränderung der Gesellschaft und des politischen Systems ausgerichtet (wie im so genannten ,,alten“ Ehrenamt), sondern vielmehr auf individuelle Selbstverwirklichung und ,systemkonforme Ökonomisierung und Issue-, Spaß- und Event-Orientierung der politischen Beteiligung “ (Baringhorst 2015, S. 17) im Sinne einer Konsumhaltung. Damit einher geht auch eine Zunahme solcher Protestformen, die niedrigschwellig und von Spontanität geprägt sind, zum Beispiel flashmobs oder sit ins wie sie auch im Rahmen der occupy-Bewegung häufig zum Einsatz kamen (Kavada 2014), bei denen neue soziale Medien eine zentrale Rolle spielen und die sich meist auf einzelne Themen beschränken.

$\mathrm{Ob}$ nun in direkter Reaktion auf die Veränderungen der Motivation und im Protestverhalten oder nicht, bieten NGOs, so die Bewegungs- und Protestforschung, interessierten Bürger*innen inzwischen oftmals vielfältige Möglichkeiten der Beteiligung an, die jede/r einzelne dann entsprechend der eigenen Interessen und Motivation 
konsumieren kann. Ebenso wie im Fall der Mobilisierung von Protesten erleichtern Webseiten und soziale Medien es den NGOs ihr Angebot entsprechend zu diversifizieren (Bennett und Segerberg 2011). Webseiten und Plattformen wie Twitter und Instagram erlauben es NGOs dabei, Menschen nicht nur allgemein zum beispielsweise Umweltschutz aufzufordern und ihre Kampagnen zu amplifizieren, wie Hall et al. (2020, S. 162) generell feststellen und wie es ein Senior Media Analyst von Greenpeace bereits 2009 konstatierte (Lorenzen zitiert in DeLuca 2009). Sondern sie erlauben es auch, spezifische Handlungsaufrufe zu verbreiten (Hall et al. 2020, S. 162; siehe auch Waters und Jones 2011, S. 254). Diese können sowohl auf bestimmte Themen als auch auf unterschiedliche Anhänger*innengruppen ausgerichtet und somit kundenspezifisch sein. NGOs können auf diesen Wegen zum Beispiel zum Kauf oder Boykott eines bestimmten Produktes aufrufen, zur Unterschrift unter eine bestimmte Petition, zur Teilnahme an einer bestimmten Demonstration etc.

„Kundenspezifische“ Botschaften werden aber nicht nur an individuelle Spender*innen und Unterstützter*innen gerichtet, sondern auch an institutionelle Geber, wie Ergebnisse der IB-Forschung zu NGOs mit Blick auf Strukturen zeigen. Dabei seien NGOs nach Ansicht von Tallberg und Uhlin (2011, S. 124) um institutionelle Geber weit mehr bemüht als um Hilfsbedürftige. Auch Monika Krause konstatiert marktähnliche Beziehungen zwischen humanitären NGOs und ihren institutionellen Gebern, die zum Beispiel darin zum Ausdruck kommen, dass Erstere für Letztere „Projekte in einem Quasimarkt produzieren“ (Krause 2014, S. 4). Dadurch werden Geber von NGOs als Konsument*innen betrachtet (Krause 2014, S. 4). Ähnlich klingt es auch in einem Beitrag des Leiters Marketing der deutschen Sektion von Handicap International: „Die Positionierung eines Produkts oder einer Dienstleistung im Markt sollte immer ein bestimmtes Nutzenversprechen, den sogenannten Consumer Benefit, beinhalten, der bewusst auf die Wünsche und Bedürfnisse der Kunden abzielt. Wenn man aus Marketingsicht Kunden von gemeinnützigen Organisationen in ,Spender und Förderer' übersetzt, könnte man dort analog von ,Donor Benefit' sprechen. Dieser kann im Fall von Handicap International wie folgt formuliert werden: Gemeinsam mit Handicap International kann ich Lebensbedingungen von Menschen mit Behinderung verbessern und Risiken verhindern, die zu Behinderung führen“ (Schiffelmann 2018, S. 131). Die marktähnlichen Beziehungen in der humanitären Hilfe manifestieren sich nicht zuletzt im Verhältnis der NGOs zu den Hilfsempfänger*innen, die nicht nur als Konsument*innen oder als Klient*innen betrachtet würden, sondern selbst als „Ware“ (Krause 2014, S. 4).

Die einzelnen genannten Forschungsstränge eint die Konstatierung einer Kommerzialisierung von NGOs, darüber hinaus jedoch besteht Uneinigkeit über einerseits die Kausalzusammenhänge mit Blick auf die Gründe für Kommerzialisierung und andererseits die Folgen der Kommerzialisierung.

\section{Triebfedern für Kommerzialisierung}

Ein Forschungsdesiderat betrifft die Kausalzusammenhänge der oben genannten Entwicklungen mit Blick auf Triebfedern für Kommerzialisierung. Ein Teil der Literatur sieht zum Beispiel die Professionalisierung der NGOs in der zunehmenden Stan- 
dardisierung ihrer Arbeit und dem zunehmenden Wettbewerb der Organisationen untereinander begründet: Für bestimmte Tätigkeiten, wie das Marketing oder das Controlling, aber auch das Einwerben von Spendengeldern, bedarf es spezialisierter Kenntnisse und Kompetenzen (Frantz und Martens 2006: 62), die sich dann in den Stellenprofilen der Mitarbeiter*innen niederschlagen. Andere sehen wiederum Hinweise für entgegengesetzte Kausalzusammenhänge und Pfadabhängigkeiten. Demnach brächten die hauptamtlichen NGO-Mitarbeiter*innen aufgrund ihrer Ausbildung ein gewisses ,mindset“ in die Organisation mit, das eher dem von Unternehmensmanager*innen entspricht und das dem unternehmerischen Modell eine Überlegenheit gegenüber anderen zuschreibt (Thomas 2013, S. 22; siehe auch Sturdy und Grey 2003). Dieses mindset käme nicht zuletzt in der Ausrichtung an bestimmten „,marktorientierten“ Einstellungen und Kapazitäten (du Gay et al. 1996, S. 267; siehe auch Maier und Meyer 2011, S. 738) wie „Exzellenz“, „Innovation“, „Flexibilität“" zum Ausdruck (du Gay et al. 1996, S. 266, 269, 274, 277). Dies fördere eine zunehmende Standardisierung, Korporatisierung und Kommodifizierung. Um zu untersuchen, wie stark Mitarbeitende von NGOs bereits vor ihrer Tätigkeit über ein entsprechendes mindset verfügten, könnten qualitative Interviews mit diesen Mitarbeitenden zum Zeitpunkt des erstmaligen Arbeitsbeginns in einer NGO und zu einem späteren Zeitpunkt, nach einer gewissen Dauer der Beschäftigung, erfolgen. Hingegen könnte eine Analyse der Biographien der NGO-Mitarbeitenden, ihrer Sozialisation in Universitäten und/oder ihre vorherige Beschäftigung bei anderen Arbeitgebern Aufschluss über die Relevanz der Ausbildung von NGO-Mitarbeitenden für eine marktorientierte Einstellung geben. Vergleichbare Einstellungen bei unterschiedlicher Sozialisation könnten ein Hinweis sein, dass statt äußerer Einflüsse vielmehr die internen Organisationsprozesse und -strukturen ausschlaggebend sind, ob und in welchem Maße Mitarbeitende eine kommerzielle Sichtweise in Bezug auf ihre Tätigkeit entwickeln.

Wiederum andere sehen den Wettbewerb der NGOs um Gelder sowie entsprechende kompetitive Strukturen (Maier et al. 2016, S. 72-73) als Triebfeder für Kommerzialisierung. Um ihre Glaubwürdigkeit zu beweisen und Gelder zu erhalten, aber auch um sich von anderen NGOs abzugrenzen, adaptierten NGOs demnach vermehrt organisationsinterne Kodizes, unterzögen sich freiwillig einem regelmäßigen Monitoring durch Außenstehende oder veröffentlichten Rechenschaftsberichte (Jordan und van Tuijl 2006; Brown 2008; Roberts et al. 2005, S. 1849). Des Weiteren könnte eine Triebfeder für Kommerzialisierung auch in der Beratungsindustrie liegen, die sich um NGOs herum gebildet hat. Ähnlich wie Unternehmen greifen auch NGOs auf entsprechende Unterstützung zurück (Joachim und Schneiker 2018a) und lassen sich zum Beispiel mit Blick auf die Einwerbung von Fördermitteln von der EU, die Neugestaltung der Organisationsstruktur oder die Gestaltung der Projektarbeit beraten. Dies kann Korporatisierung und/oder organisationsinterne Rationalisierungsprozesse befördern. Studien, die zum Beispiel mittels process tracing die Entwicklung von NGOs nachzeichnen, könnten Aufschluss auf die Rolle externer Berater*innen und Erfordernissen von Gebern geben. Dabei könnte man auch NGOs vergleichen, die unterschiedlich stark von staatlichen Gebern abhängen sowie solche, die sich beraten lassen und solche, die sich nicht beraten lassen. 
Schließlich wurde in der Literatur zur Kommerzialisierung des Non-Profit-Sektors kaum auf Entwicklungen im For-Profit-Sektor eingegangen. Doch die Anpassung von NGOs an Unternehmen ist keine Einbahnstraße, sondern auch Unternehmen können sich NGOs angleichen, wie die IB-Forschung zu NGOs zeigt. Diese Entwicklungen können sich gegenseitig bedingen. Viele Unternehmen haben zum Beispiel gemeinwohlorientierte Standards wie Menschenrechte oder Regeln zum Umweltschutz übernommen und eine corporate-social-responsibility-Politik und entsprechende Strukturen und Prozesse etabliert. Darüber hinaus engagieren sich einige Unternehmen auch karitativ, indem sie zum Beispiel eigene Stiftungen gegründet haben. Einige Autor*innen sprechen in diesem Kontext von „Philanthrokapitalismus“ (s. z. B. Hopgood 2008). Insbesondere im humanitären Feld führen zunehmend auch Unternehmen Aufgaben durch, die vormals exklusiv von NGOs durchgeführt wurden (Joachim und Schneiker 2012). Teilweise werden Unternehmen von institutionellen Geldgebern bei der Projektvergabe sogar bevorzugt, da sie aufgrund ihrer unternehmerischen Tätigkeiten in den Einsatzländern, so zumindest die Begründung, über effektivere Netzwerke und Strukturen verfügen als NGOs. So erklärt der Gründer der NGO Islamic Relief: „In the past, the money used to go to the specialised humantarian organisations for emergency response and development. Now it goes to private companies who are efficient in doing development work as a business." (ElBanna 2018, S. 7) Unternehmen werden teilweise auch deshalb bevorzugt, weil sie weniger von normativen Kriterien geleitet agieren würden. So konstatiert Spearin im Fall von Sicherheitsunternehmen, die für Regierungen humanitäre Aufgaben durchführen, dass diese im Unterschied zu auf Neutralität, Unabhängigkeit und Unparteilichkeit bedachten NGOs keine Berührungsängste mit militärischen Akteuren hätten und sich eher in militärische Strukturen einbeziehen ließen (Spearin 2008, S. 363). Dabei werden Unternehmen unmittelbar zu Mitbewerbern von NGOs um Gelder und Projekte. In welchem Maße die oben dargelegten Tendenzen der Standardisierung, Rationalisierung oder der Professionalisierung der neuartigen Konkurrenz geschuldet ist, gilt es noch zu bestimmen. Zum Beispiel könnte man eine Netzwerk-Analyse durchführen und dadurch bestimmen, wie sich das Umfeld und die Beziehungen der Non-Profit-Organizationen zu For-Profit-Organisationen verändert haben. Ein Vergleich der Internetseiten von Sicherheitsunternehmen mit jener von humanitären NGOs zeigt zumindest schon einmal, dass sich die Selbstdarstellungen kaum mehr voneinander unterscheiden (Joachim und Schneiker 2012).

Doch nicht nur zu den Triebfedern, auch zu den Folgen der Kommerzialisierung gibt es unterschiedliche Ansichten und Blindstellen in der Literatur.

\section{Folgen der Kommerzialisierung}

Die Folgen der Kommerzialisierung können die Art und Qualität der geleisteten Hilfe und ihre Reichweite betreffen. Einerseits wird auf die positiven Effekte einer kompetitiven und transparenten Vergabepraxis durch institutionelle Geber wie Ministerien hingewiesen. Nur so könne, so zum Beispiel der Leiter des Bereiches humanitäre Hilfe von Oxfam Deutschland e. V. in einer Stellungnahme für den Ausschuss für Menschenrechte und humanitäre Hilfe des Deutschen Bundestages, ,si- 
chergestellt werden, dass (1) die Hilfe bedarfsgerecht eingesetzt wird (2) der jeweils am besten geeignete Partner den Zuschlag bekommt und (3) die Förderung nicht von anderweitigen Interessen beeinflusst wird“ (Prangl 2019, S. 11). Andererseits weisen Studien auf die negativen Folgen des Wettbewerbes um Gelder und Projekte hin. Während sich diese unterschiedlichen Einschätzungen aus Sicht von Maier et al. (2016, S. 75-76) in der Forschung zu Non-Profit-Organisationen ungefähr die Waage halten, überwiegen gemäß der IB-Forschung zu NGOs die negativen Folgen. Denn ein Wettbewerb erzeuge nicht nur einen Erfolgsdruck für NGOs, insbesondere dann, wenn die Vergabe neuer Gelder für neue Projekte an die erfolgreiche Durchführung vergangener Projekte gekoppelt wäre. Er hätte im Falle humanitärer Hilfe auch möglicherweise zur Folge, dass das Kriterium der Bedürftigkeit durch das der Wirtschaftlichkeit verdrängt würde. So resümieren zum Beispiel Cooley und Ron auf Basis ihrer Studie über humanitäre NGOs in den Flüchtlingslagern in Goma in den 1990er-Jahren, dass NGOs ihre Arbeit dort fortgesetzt hatten, obwohl sie wussten, dass die von ihnen geleistete Hilfe von Gewaltakteuren zweckentfremdet wurde. Dabei handelten die Organisationen aus finanziellen Motiven, denn sie befürchteten, dass andere NGOs ihren Platz und die damit verbundenen Fördergelder übernehmen würden, wenn sie sich selbst zurückzögen (Cooley und Ron 2002). Des Weiteren haben Studien herausgefunden, dass humanitäre NGOs, die in Kontexten bewaffneter Konflikte arbeiten, teilweise Bedrohungen gegen oder Angriffe auf sie verschweigen, aus Sorge Geber könnten daraufhin den Geldhahn zudrehen, in Kauf nehmend, dass Mitarbeiter*innen der Organisationen Opfer von Angriffen werden (Donini 2009, S. 8; Schneiker 2018, S. 119). Andere Autor*innen haben festgestellt, dass Organisationen auch dazu neigten, Misserfolge im Zuge ihrer Projekte zu verdecken oder zu verschweigen, da sie positive Ergebnisse ihrer Arbeit vorweisen müssen, um neue Verträge von Gebern zu erhalten (Edwards 1997, S. 70; Huismann 2012; Van Brabant 2001, S. 189). Dass Wettbewerb um Gelder zu suboptimalen und gar unerwünschten Effekten führt, liegt jedoch nicht unbedingt an der kompetitiven Vergabepraxis an sich, sondern an deren Ausgestaltung. So wäre auch denkbar, dass Gelder nur an solche NGOs vergeben werden, die nach bestimmten ethischen Kriterien handeln. Das setzt aber eine andere Form der Erfolgsmessung voraus, die nicht auf Effektivität und Effizienz basiert. Entsprechend müsste die Rationalisierungsdimension der Kommerzialisierung mit Blick auf die Standardisierung an anderen Kriterien ausgerichtet sein als bisher. Untersuchungen über die Vergabepraxis von Geldern und Projekten an NGOs auf Basis vorheriger Arbeit der NGOs bzw. Aussagen darüber, ob NGOs neue Gelder aufgrund einer bestimmten vorherigen Performanz erhalten, sind jedoch schwierig, da entsprechende Daten in der Regel nicht öffentlich zugänglich sind.

Die Folgen der Kommerzialisierung betreffen möglicherweise auch das Selbstverständnis der Organisationen und ihrer Mitarbeiter*innen, doch durch die getrennte Betrachtung der unterschiedlichen Dimensionen von Kommerzialisierung ist hierüber bisher wenig bekannt und bestehende Forschung hierzu kommt vorwiegend aus dem Bereich der Non-Profit-Organisationen. Während der Prozess von einigen als reiner Transfer von Praktiken aus dem For-Profit-Sektor in den Non-Profit-Sektor (Bromley und Meyer 2014, S. 1) und damit als ein eher technischer und unpolitischer Vorgang begriffen wird, gehen andere von weitreichenderen Folgen aus. So 
vermuten beispielsweise Albert und Whetten (1985, S. 272), dass eine Kommerzialisierung in einer NGO einen ,struggle ... over the very soul of the institution“ bedingt und Sanders und McClellan (2014, S. 69) sehen durch die Kommerzialisierung die Fähigkeit von NGOs bedroht, sich durch die Adressierung sozialer Probleme von anderen Sektoren abzugrenzen. Denn ,firm-like“ geht nach Auffassung dieser Autoren mit veränderten „Normen oder ,Mentalitäten“" einher (Abrahamsen und Williams 2009, S. 3) und einem ,mind-set“, welches in solchen Praktiken und Diskursen zum Ausdruck käme, die einer wirtschaftlichen Rationalität folgen (Leander und van Munster 2007, S. 204). Weil Evaluationen und Rechenschaftspflichten von NGOs verlangen, ihr Handeln und ihre Ergebnisse messbar zu machen (Hwang und Powell 2009, S. 269) und mittels „Erfolgsindikatoren“ (Deutsche Welthungerhilfe e. V. 2019, S. 27) zu bestimmen, kann dies nach Auffassung von Leander und van Munster disziplinierende Effekte auf die Mitarbeiter*innen der Organisationen haben (Leander und van Munster 2007, S. 209), die, in Antizipation dieser Rechenschaftspflicht, ihr Verhalten angleichen (du Gay et al. 1996, S. 271). Ähnlich wie bei der Frage nach den Triebfedern für Kommerzialisierung könnten Studien über die Entwicklung von NGOs sowie Vergleiche zwischen NGOs, die zu unterschiedlichen Zeitpunkten und in unterschiedlicher Stärke Strukturen und Prozesse aus dem For-Profit-Sektor übernommen haben, Aufschluss über die Folgen von Kommerzialisierung geben.

Doch inwiefern welche Dimension von Kommerzialisierung das Selbstverständnis von NGOs verändert und inwiefern es hier ggf. Unterschiede bei den NGOs gibt, ist empirisch unterforscht. So ist unklar, ob folgende Aussage von Manfred Glagow aus dem Jahr 1993 heute noch zutrifft: „In Deutschland sind die harten medien-professionellen Vorgehensweisen amerikanischer oder aus den USA stammender NROs höchst umstritten. Insbesondere im Bereich der Hilfe für Kinder in der Dritten Welt wird von einigen NROs mit hochgradig emotionalisierten Anzeigen, die z. T. sogar von falschen Angaben und Voraussetzungen leben, um Spenden gekämpft. Damit wird aber das zumindest in der Bundesrepublik Deutschland noch vorhandene gemeinsame Ethos der NRO als nicht quasi-marktorientierte Händler in Sachen Caritas verletzt" (Glagow 1993, S. 317). Zu bestimmen wäre somit, ob sich Kommerzialisierung auch durch kontextbedingte Pfadabhängigkeiten erklären lässt, für die NGOs aufgrund ihrer kulturellen Vorprägung anfälliger sind als andere. Länder- und Sektorenübergreifende Vergleiche von NGOs und ihren Entwicklungen mittels process tracing könnten helfen, diese Forschungslücke zu schließen.

Wenn sich NGOs auch mit Blick auf ihr Selbstverständnis immer mehr Unternehmen annähern, dann stellt sich die Frage, ob für das vormals von NGOs repräsentierte solidarische und gemeinwohlorientierte, selbstlose Engagement in der Gesellschaft kein Bedarf mehr ist oder ob dieses von anderen Akteuren besetzt wird. Auf Letzteres deutet die Forschung zum politischen Protest und zum Ehrenamt hin, die untersucht, warum so genannte Hybrid-Organisationen wie Campact, die online- und offlineAktivitäten anbieten, erfolgreich sind: „Die hohe Zahl von Personen, die sich an den Aktionen der Hybrid-Organisationen beteiligen, zeigt, dass es durchaus einen Bedarf für politische Beteiligungs- und Engagementmöglichkeiten außerhalb fester, verbindlicher Organisationsstrukturen gibt.“ (Voss 2013, S. 220) Zudem scheinen soziale Bewegungen wie Fridays for Future (FFF) nun den Platz zu besetzen, den vormals NGOs eingenommen haben. Das Problem, mit dem sich FFF befasst - der 
Klimawandel - ist ja weder ein neues Thema noch eines, das bislang nicht von NGOs behandelt worden wäre. Organisationen wie Greenpeace befassen sich seit langer Zeit damit. Dennoch sind diejenigen, die jetzt Teil von FFF sind, nicht im Rahmen etablierter NGOs wie Greenpeace aktiv geworden, sondern haben FFF ,gegründet" und/oder engagieren sich im Rahmen dieser sozialen Bewegung. Studien über die Entwicklung von Mitgliederzahlen von NGOs sowie über die Entwicklung der Stärke des Engagements von Mitgliedern könnten Hinweise darauf geben, inwiefern Bewegungen wie FFF eine Konkurrenz für NGOs sind.

Des Weiteren kann Kommerzialisierung auch Auswirkungen auf die Beziehungen zwischen Hauptamtlichen und Ehrenamtlichen haben. Bisher hat die Forschung diese Beziehungen vor allem als Konflikt zwischen wirtschaftlichen Handlungsmaximen (Hauptamtliche) einerseits und Solidarität und Altruismus (Ehrenamtliche) andererseits behandelt (Frantz und Martens 2006: 66-67). Berücksichtigt man jedoch, dass auch Ehrenamtliche - im oben dargelegten Sinne der Kommerzialisierung - von eigennützigen Motiven geleitet sein können, basieren Konflikte zwischen Freiwilligen und Hauptamtlichen möglicherweise auf einem anderen Spannungsverhältnis. Statt dem Konflikt zwischen Organisationsnutzen (mehr Sichtbarkeit, mehr Projekte, mehr Gelder, Organisationswachstum) und Nutzen für die Allgemeinheit (Hilfe für Bedürftige) geht es möglicherweise vielmehr um den Konflikt zwischen Organisationsnutzen und Nutzen für den/die Einzelne/n (Selbstverwirklichung). Entsprechende Konflikte und Spannungen können mittels Umfragen und qualitativen Interviews oder ggf. teilnehmender Beobachtung untersucht werden.

Vor diesem Hintergrund muss gefragt werden, ob es unter den NGOs auch zu einem Wettbewerb um Freiwillige kommt und welche NGOs dabei Vorteile gegenüber anderen haben. Erhalten diejenigen NGOs die meiste Unterstützung von Freiwilligen, die ein möglichst diversifiziertes Angebot zum Engagement machen oder diejenigen NGOs, die es Freiwilligen am besten ermöglichen, ihr Engagement für alle sichtbar und im Sinne des Eigennutzes optimal darzustellen? Reicht es noch aus für eine NGO, sich einer "guten Sache“ zu verschreiben, um Spendengelder zu erhalten? Oder muss eine NGO nicht vielmehr eine Nische entwickeln, sowohl in der Sache, für die sie agiert, als auch in der Art und Weise, wie sie dies tut? Viel grundsätzlicher ist zu fragen, ob und in welchem Maße ein solches Nischendasein möglich ist. Denn eine weitere mögliche und paradoxe Folge der Kommerzialisierung sind isomorphe Tendenzen. In dem Maße, wie NGOs versuchen, sich aus der Zahl ihrer Mitbewerber hervorzuheben um sich gegenüber institutionellen aber auch individuellen Geldgebern einen Vorteil zu verschaffen, führen die hierfür unternommenen Schritte wie Korporatisierung oder Standardisierung zur Angleichung unter den Organisationen (Frumkin und Galaskiewicz 2004) und lassen die Unterschiede nur noch schwerlich erkennen. Gleichzeitig kann dieser Trend auch die bereits bestehenden Machtasymmetrien unter den NGOs verstärken (Dany 2014). Nicht alle NGOs sind schon allein aus finanziellen und personellen Gründen gleichermaßen in der Lage Firmen ähnliche Strategien zu verfolgen oder lehnen diese möglicherweise und wie bereits oben angedeutet aufgrund ihres Selbstverständnisses gänzlich ab (Glagow 1993, S. 317). 


\section{Zusammenfassung und Ausblick}

Das Phänomen der Kommerzialisierung von NGOs ist vielschichtig und erfasst nicht nur organisationsinterne Strukturen und Prozesse, sondern auch das Außenhandeln der NGOs und ihre Beziehungen zu andere Akteuren. Da unterschiedliche Teildisziplinen der Politikwissenschaft aber meist nur einzelne Aspekte der Kommerzialisierung untersuchen, wird das Phänomen in der Regel nicht in seiner Gesamtheit erfasst. Daher haben wir in diesem Aufsatz unterschiedliche Forschungsstränge zu Kommerzialisierung zusammengetragen und eine Typologie von Kommerzialisierung vorgeschlagen, die unterschiedliche Dimensionen des Phänomens erfasst.

Neben den noch unzureichend beforschten Gründen und Folgen der Kommerzialisierung für NGOs selbst hat diese sich abzeichnende Entwicklung im Non-ProfitSektor aber auch Auswirkungen auf die wissenschaftliche Auseinandersetzung mit der Thematik. Nach wie vor beruht ein Gros der Forschung auf der herkömmlichen Definition von NGOs als unabhängige und am Allgemeinwohl orientierten Organisationen. Es muss jedoch aufgrund der genannten Veränderungen diskutiert werden, ob dieses Verständnis noch angemessenen ist. Bis auf wenige Ausnahmen, wie der humanitären Hilfe (Barnett und Weiss 2008b; Dany 2019; van Brabant 2010), ist eine solche Diskussion jedoch bislang ausgeblieben. Zwar kann es sein, dass Kommerzialisierung nicht in allen Politikfeldern gleichermaßen auftritt, doch haben die oben angeführten Beispiele gezeigt, dass Kommerzialisierung bei NGOs aus unterschiedlichen Bereichen vorzufinden ist. Darüber hinaus ist die Kommerzialisierung von NGOs auch für andere Forschungsbereiche als die NGO-Forschung relevant, zum Beispiel für die Governance-Forschung. Während diese bereits eingehend die Sinnhaftigkeit der sektoralen Unterscheidung zwischen privat und öffentlich hinterfragt (Abrahamsen und Williams 2009; Berndtsson und Stern 2011), wird die Grenze zwischen dem privaten For-Profit- und dem privaten Non-Profit-Bereich jedoch als weitestgehend intakt angenommen, obgleich die Kommerzialisierung von NGOs an einer klaren Abgrenzung zweifeln lässt.

Darüber hinaus erfordert die Untersuchung der oben genannten Punkte von Forscher*innen aufwendige Forschungsdesigns, die es erlauben, das Innenleben der Organisationen sowie das Handeln einzelner Mitarbeiter*innen zu untersuchen. Dazu bedarf es nicht nur einer Abkehr von der Konzeptionalisierung von Akteuren als monolithische und einheitlich handelnde. Es bedarf auch anderer Methoden, z. B. ethnographischer, wie teilnehmender Beobachtung, und counter-factual reasoning, um zum Beispiel zu bestimmen, inwieweit Mitarbeiter*innen nach wie vor und in ,hard cases“ sich den ursprünglichen Werten der Organisation verpflichtet sehen und basierend auf diesen handeln oder aber ihr Handeln eher an einer ökonomischen Logik ausrichten. Diese Frage ist mit der Frage nach der Verwischung sektoraler Grenzen verbunden. Ist eine kommerzialisierte NGO also noch eine NGO oder vielmehr ein als gemeinnützig deklariertes Unternehmen?

Funding Open Access funding enabled and organized by Projekt DEAL.

Open Access Dieser Artikel wird unter der Creative Commons Namensnennung 4.0 International Lizenz veröffentlicht, welche die Nutzung, Vervielfältigung, Bearbeitung, Verbreitung und Wiedergabe in jeglichem Medium und Format erlaubt, sofern Sie den/die ursprünglichen Autor(en) und die Quelle ord- 
nungsgemäß nennen, einen Link zur Creative Commons Lizenz beifügen und angeben, ob Änderungen vorgenommen wurden.

Die in diesem Artikel enthaltenen Bilder und sonstiges Drittmaterial unterliegen ebenfalls der genannten Creative Commons Lizenz, sofern sich aus der Abbildungslegende nichts anderes ergibt. Sofern das betreffende Material nicht unter der genannten Creative Commons Lizenz steht und die betreffende Handlung nicht nach gesetzlichen Vorschriften erlaubt ist, ist für die oben aufgeführten Weiterverwendungen des Materials die Einwilligung des jeweiligen Rechteinhabers einzuholen.

Weitere Details zur Lizenz entnehmen Sie bitte der Lizenzinformation auf http://creativecommons.org/ licenses/by/4.0/deed.de.

\section{Literatur}

Abrahamsen, Rita, und Michael C. Williams. 2009. Security beyond the state: global security assemblages in international politics. International Political Sociology 3:1-17.

Albert, Stuart, und David A. Whetten. 1985. Organizational identity. Research in Organizational Behavior 7:263-295.

Alexander, Jeffrey A., und Bryan J. Weiner. 1998. The adoption of the corporate governance model by nonprofit organizations. Nonprofit Management \& Leadership 8:223-242.

Almadhoun, Mahmoud. 2018. Kompetenzbasiertes Personalmanagement bei Islamic Relief Deutschland. In Strategisches Management humanitärer NGOs, Hrsg. Michael Heuser, Tarek Abdelalem, 197-210. Wiesbaden: Gabler.

ALNAP. 2018. The state of the humanitarian system. London: ALNAP/ODI.

Barakso, Maryann. 2010. Brand identity and the tactical repertoires of advocacy organizations. In Advocacy organizations and collective action, Hrsg. Aseem Prakash, Mary Kay Gugerty, 155-176. Cambridge: Cambridge University Press.

Baringhorst, Sigrid. 2015. Konsum und Lebensstile als politische Praxis - Systematisierende und historisch kontextualisierende Annährungen. Forschungsjournal Soziale Bewegungen 28:17-27.

Barnett, Michael, und Thomas G. Weiss. 2008a. Humanitarianism. A brief history of the present. In $H u$ manitarianism in question. Politics, power, ethics, Hrsg. Michael Barnett, Thomas G. Weiss, 1-48. Ithaca: Cornell University Press.

Barnett, Michael, und Thomas G. Weiss. 2008b. Humanitarianism in question. Politics, power, ethics. Ithaca: Cornell University Press.

Beher, Karin, Reinhard Liebig, und Thomas Rauschenbach. 2001. Vom Motivations- zum Strukturwandel. Analysen zum Ehrenamt in einer sich verändernden Umwelt. In Bürgerengagement in Deutschland. Bestandsaufnahme und Perspektiven, Hrsg. Rolf G. Heinze, Thomas Olk, 255-281. Opladen: Leske + Budrich.

Bennett, Lance, und Alexandra Segerberg. 2011. Digital media and the personalization of collective action. Social technology and the organization of protests against the global economic crisis. Information, Communication \& Society 14:770-799.

Berndtsson, Joakim, und Maria Stern. 2011. Private security and the public_-Private divide: contested lines of distinction and modes of governance in the Stockholm-Arlanda security assemblage. International Political Sociology 5:408-425.

Blühdorn, Ingolfur. 2013. Simulative Demokratie - Neue Politik nach der postdemokratischen Wende. Berlin: Suhrkamp.

Bromley, Patricia, und John W. Meyer. 2014. "They are all organizations": the cultural roots of blurring between the nonprofit, business, and government sectors. Administration \& Society 49:939-966.

Brown, L. David. 2008. Creating credibility. Legitimacy and accountability for transnational civil society. Sterling: Kumarian Press.

BUND. 2020. Über uns. Organisation. https://www.bund.net/ueber-uns/organisation. Zugegriffen: 30. März 2020.

Bundeszentrale für politische Bildung. o.J.. Greenpeace. https://www.bpb.de/gesellschaft/umwelt/dossierumwelt/62374/umweltorganisationen. Zugegriffen: 5. Febr. 2020.

Collinson, Sarah, und Samir Elhawary. 2012. Humanitarian space: a review of trends and issues. London: ODI, Humanitarian Policy Group. 
Cooley, Alexander, und James Ron. 2002. The NGO scramble: organizational insecurity and the political economy of transnational action. International Security 27:5-39.

Dany, Charlotte. 2014. Janus-faced NGO participation in global governance. Structural constraints for NGO influence. Global Governance. A Review of Multilateralism and International Organizations 20:419-436.

Dany, Charlotte. 2019. Exploring the political agency of humanitarian NGOs: Médecins Sans Frontières during the European refugee crisis. Global Society 33:184-200.

DeLuca, Kevin Michael. 2009. Praxis: interview. Greenpeace international media analyst reflects on communicating climate change. Environmental Communication 3:263-269.

Deutsche Welthungerhilfe e.V.. 2019. Jahresbericht 2018. https:/www.welthungerhilfe.de/aktuelles/ publikation/detail/jahresbericht-der-welthungerhilfe-2018/. Zugegriffen: 5. Febr. 2020.

Diakonie Katastrophenhilfe. 1993. Verhaltensgrundsätze für die Internationale Rot Kreuz und Roter Halbmond Bewegung sowie Nichtregierungsorganisationen (NROs) in der Katastrophenhilfe. Deutsche Fassung.

Dietrich, Fridolin. 2018. Non-Profit Branding - Markenführung in Non-Profit-Organisationen. In Strategisches Management humanitärer NGOs, Hrsg. Michael Heuser, Tarek Abdelalem, 116-122. Wiesbaden: Gabler.

Donini, Antonio. 2009. Afghanistan: humanitarianism under threat. Briefing Paper. Boston: Feinstein International Center. http://fic.tufts.edu/assets/Donini-Afghanistan-1.pdf. Zugegriffen: 9. Juli 2014.

Du Gay, Paul, Graeme Salaman, und Bronwen Rees. 1996. The conduct of management and the management of conduct: contemporary managerial discourse and the constitution of the "competent" manager. Journal of Management Studies 33:263-282.

DZI. 2020. Spenden-Siegel. https://www.dzi.de/spenderberatung/das-spenden-siegel/. Zugegriffen: 30. März 2020.

Eberwein, Wolf-Dieter, und Sabine Saurugger. 2013. The professionalization of international non-governmental organizations. In Routledge handbook of international organization, Hrsg. Bob Reinalda, 257-269. London: Routledge.

Edwards, Michael. 1997. Becoming a learning organization, or, the search for the Holy Grail? In Strategies of public engagement: shaping a Canadian agenda for international co-operation, Hrsg. David W. Gillies, 63-94. Montreal: McGill-Queen's University Press.

El-Banna, Hany. 2018. "Our Challenge is to Change the Climate of Humanitarian Work"-The Future Role of NGOs in Civil Societies. Interview. In Strategisches Management humanitärer NGOs, Hrsg. Michael Heuser, Tarek Abdelalem, 3-14. Wiesbaden: Gabler.

Frantz, Christiane. 2010. Nichtregierungsorganisationen (NGOs) als internationale Akteure. In Einführung in die Politikwissenschaft, Hrsg. Christiane Frantz, Klaus Schubert, 190-1999. Münster: LIT.

Frantz, Christiane, und Kerstin Martens. 2006. Nichtregierungsorganisationen (NGOs). Springer VS.

Frumkin, Peter, und Joseph Galaskiewicz. 2004. Institutional isomorphism and public sector organizations. Journal of Public Administration Research and Theory 14:283-307.

Glagow, Manfred. 1993. Die Nicht-Regierungsorganisationen in der internationalen Entwicklungszusammenarbeit. In Handbuch der Dritten Welt, Hrsg. Dieter Nohlen, Franz Nuscheler, 304-326. Bonn: JHW Dietz.

Götz, Norbert. 2011. Civil society and NGO: far from unproblematic concepts. In The Ashgate companion of nonstate actors, Hrsg. Bob Reinalda, 185-208. Farnham: Ashgate.

Gourevitch, Peter A., und David A. Lake. 2012. Credibility and compromises. In The credibility of transnational NGOs. When virtue is not enough, Hrsg. Peter A. Gourevitch, David A. Lake, und Janice Stein Gross, 193-207. Cambridge: Cambridge University Press.

Gross Stein, Janice. 2008. Humanitarian organizations. Accountable-Why, tho whom, for what, and how? In Humanitarianism in question: politics, power, ethics, Hrsg. Michael Barnett, Thomas G. Weiss, 124-142. Ithaca: Cornell University Press.

Hall, Nina, Hans Peter Schmitz, und J. Michael Dedmon. 2020. Transnational advocacy and NGOs in the digital era: new forms of networked power. International Studies Quarterly 64:159-167.

Han-Broich, Misun. 2012. Ehrenamt und Integration. Die Bedeutung sozialen Engagements in der (Flüchtlings-)Sozialarbeit. Wiesbaden: Springer VS.

Hansmann, Henry B. 1980. The role of nonprofit enterprise. Yale Law Journal 89(5):835-902.

Heins, Volker. 2002. Weltbürger und Lokalpatrioten. Eine Einführung in das Thema Nichtregierungsorganisationen. Opladen: Leske + Budrich.

Heins, Volker. 2008. Nongovernmental organizations in international society: struggles over recognition. New York: Palgrave Macmillan. 
Heuser, Michael, und Tarek Abdelalem. 2018. Vorwort. Strategisches Management humanitärer NGOs Die Professionalisierung nimmt zu. In Strategisches Management humanitärer NGOs, Hrsg. Michael Heuser, Tarek Abdelalem, v-vi. Wiesbaden: Gabler.

Hopgood, Stephen. 2008. Saying "No" to Wal-Mart? Money and morality in professional humanitarianism. In Humanitarianism in question: politics, power, ethics, Hrsg. Michael Barnett, Thomas G. Weiss, 98-123. Ithaca: Cornell University Press.

Horwitz, Melvin. 1988. Corporate reorganization: the last gasp or last clear chance for the tax-exempt, nonprofit hospital? American Journal of Law \& Medicine 13:527-559.

Huismann, Wilfried. 2012. Schwarzbuch WWF. Dunkle Geschäfte im Zeichen des Panda. Gütersloh: Gütersloher Verlagshaus.

Hwang, Hokyu, und Walter W. Powell. 2009. The rationalization of charity: the influences of professionalism in the nonprofit sector. Administrative Science Quarterly 54:268-298.

Joachim, Jutta. 2020. NGOs in world politics. In The globalization of world politics, Hrsg. John Baylis, Steve Smith, und Patricia Owen, 349-364. Oxford: Oxford University Press.

Joachim, Jutta, und Andrea Schneiker. 2012. New humanitarians? Frame appropriation through private military and security companies. Millennium: Journal of Internationals Studies 40:365-388.

Joachim, Jutta, und Andrea Schneiker. 2018a. Private security and identity politics. Ethical hero warriors, professional managers and new humanitarians. London: Routledge.

Joachim, Jutta, und Andrea Schneiker. 2018b. Humanitarian NGOs as Businesses and Managers: Theoretical Reflection on an Under-Explored Phenomenon. International Studies Perspectives 19:170-187.

Jordan, Lisa, und Peter van Tuijl (Hrsg.). 2006. NGO accountability: politics, principles and innovations. London: Routledge.

Kavada, Anastasia. 2014. Creating the collective: social media, the occupy movement and its constitution as a collective actor. Information, Communication \& Society 18:872-886.

Krause, Monika. 2014. The good project: humanitarian relief NGOs and the fragmentation of reason. Chicago: University of Chicago Press.

Lang, Sabine. 2013. NGOs, civil society and the public sphere. Cambridge: Cambridge University Press.

Leander, Anna, und Rens van Munster. 2007. Private security contractors in the debate about Darfur: reflecting and reinforcing neo-liberal governmentality. International Relations 21:201-216.

Lundström, Tommy. 2001. Child protection, voluntary organizations, and the public sector in Sweden. Voluntas: International Journal of Voluntary and Nonprofit Organizations 12:355-371.

Maier, Florentine, und Michael Meyer. 2011. Managerialism and beyond: discourses of civil society organization and their governance implications. Voluntas: International Journal of Voluntary and Nonprofit Organizations 22:731-756.

Maier, Florentine, Michael Meyer, und Martin Steinbereithner. 2016. Nonprofit organizations becoming business-like: a systematic review. Nonprofit and Voluntary Sector Quarterly 45:64-86.

More-Hollerweger, Eva. 2014. Entwicklungen von Freiwilligenarbeit. In Forschung zu Zivilgesellschaft, NPOs und Engagement. Quo Vadis?, Hrsg. Annette Zimmer, Ruth Simsa, 301-314. Wiesbaden: Springer VS.

PeTA. 2020. PeTA store. https://www.petastore.de/. Zugegriffen: 5. Febr. 2020.

Prangl, Wolfgang. 2019. Fragenkatalog zur Anhörung des Ausschusses für Menschenrechte und humanitäre Hilfe zum Bericht der Bundesregierung über die deutsche humanitäre Hilfe im Ausland 2014-2017 (BT-Drs. 19/5720) am 20. März 2019. Ausschussdrucksache 19(17)38. https://www. google.com/url?sa=t\&rct=j\&q=\&esrc=s\&source=web\&cd=\&ved=2ahUKEwiNh-WJluvpAhVMTB UIHQovDx0QFjABegQIChAB\&url=https $\% 3 \mathrm{~A} \% 2 \mathrm{~F} \% 2 \mathrm{Fwww}$.bundestag.de $\% 2$ Fresource $\% 2 \mathrm{Fblob} \%$ 2F632042\%2F773f043b3cce6b3a1f6dd3cf5d0a65fe\%2Fstellungnahme_prangl-data.pdf\&usg=AOv Vaw3Fpy-FV08LzHq-fHINGI78. Zugegriffen: 5. Juni 2020.

Pratt, Brian, Jerry Adams, und Hannah Warren. 2006. Official agency funding of NGOs in seven countries: mechanisms, trends and implications. INTRAC occasional papers 46. Oxford: International NGO Training and Research Centre (INTRAC). http://www.intrac.org/data/files/resources/407/OPS46-Official-Agency-Funding-of-NGOs-in-Seven-Countries.pdf. Zugegriffen: 9. Juli 2014.

Priller, Eckhard. 2011. Dynamik, Struktur und Wandel der Engagementforschung: Rückblick, Tendenzen und Anforderungen. In Zivilengagement. Herausforderungen für Gesellschaft, Politik und Wissenschaft, Hrsg. Eckhard Priller, Mareike Alscher, Dietmar Dathe, und Rudolph Speth, 11-40. Berlin: LIT.

Rahmann, Ute. 1999. Freiwilliges Soziales Engagement im Wandel. Möglichkeiten und Grenzen der Förderung auf kommunaler Ebene. Aachen: Shaker.

Riddell, Roger C. 2008. Does foreign aid really work? Oxford: Oxford University Press. 
Roberts, Susan M., John Paul Jones III, und Oliver Fröhling. 2005. NGOs and the globalization of managerialism. World Development 33:1845-1864.

Salamon, Lester M., und Sokolowski S. Wojciech. 2016. Beyond nonprofits: re-conceptualizing the third sector. Voluntas: International Journal of Voluntary and Nonprofit Organizations 27(4):1515-1545.

Sanders, Mathew L., und John G. Mcclellan. 2014. Being business-like while pursuing a social mission: acknowledging the inherent tensions in US nonprofit organizing. Organization 21:68-89.

Schiffelmann, Thomas. 2018. Non-Profit-Branding/Markenführung in Non-Profit-Organisationen-Praxisbeispiel Handicap International. In Strategisches Management humanitärer NGOs, Hrsg. Michael Heuser, Tarek Abdelalem, 123-132. Wiesbaden: Gabler.

Schneiker, Andrea. 2018. Risk-aware or risk-averse? Challenges in implementing security risk management within humanitarian NGOs. Risk, Hazards \& Crisis in Public Policy 9:107-131.

Settele, Karin. 2018. Projektmanagement in internationalen humanitären Organisationen: Herausforderungen in einem dynamischen Umfeld. In Strategisches Management humanitärer NGOs, Hrsg. Michael Heuser, Tarek Abdelalem, 77-93. Wiesbaden: Gabler.

Spearin, Christopher. 2008. Private, armed and humanitarian? States, NGOs, international private security companies and shifting humanitarianism. Security Dialogue 39:363-382.

Strachwitz, Rupert. 2000. Management und Nonprofit-Organisationen - von der Vereinbarkeit von Gegensätzen. In Management in Nonprofit-Organisationen, Hrsg. Stefan Nährlich, Annette Zimmer, 23-36. Opladen: Leske + Budrich.

Sturdy, Andrew, und Christopher Grey. 2003. Beneath and beyond organizational change management: exploring alternatives. Organization 10:651-662.

Tallberg, Jonas, und Anders Uhlin. 2011. Civil society and global democracy. An assessment. In Global democracy: normative and empirical perspectives, Hrsg. Daniele Archibugi, Mathias Koenig-Archibugi, und Raffaele Marchetti, 211-232. Cambridge: Cambridge University Press.

Thaut, Laura, Janice Stein Gross, und Michael Barnett. 2012. In defense of virtue: credibility, legitimacy dilemmas, and the case of islamic relief. In The credibility of transnational NGOs: when virtue is not enough, Hrsg. Peter A. Gourevitch, David A. Lake, und Janice Stein Gross, 137-164. Cambridge: Cambridge University Press.

Thomas, Adrien. 2013. Towards the managerialization of trade unions? Recent trends in France and Germany. European Journal of Industrial Relations 19:21-36.

Van Brabant, Koenraad. 2001. Organizational and institutional learning in the humanitarian sector. In The charitable impulse: NGOs and development in east and north-east Africa, Hrsg. O. Barrow, M. Jennings, 183-199. Oxford, Bloomfield: James Currey und Kumarian Press.

Van Brabant, Koenraad. 2010. Managing Aid Agency Security in an Evolving World: The Larger Challenge. http://reliefweb.int/sites/reliefweb.int/files/resources/EAA88FFC127E6E7E852577EC00712 F7D-EISF_Agency_Security_nov2010.pdf. Zugegriffen: 7. Juni 2016.

Van Ham, Peter. 2008. Place Branding: The State of the Art. Annals of the American Academy of Political and Social Science 616:126-49.

Vestergaard, Anne. 2008. Humanitarian branding and the media: the case of Amnesty International. Copenhagen business school working paper. http://openarchive.cbs.dk/bitstream/handle/10398/6965/wp81\%20humanitarian $\% 20$ branding\%20\%26\%20the\%20media.pdf?sequence $=1$. Zugegriffen: 7 . Juni 2016.

Voss, Kathrin. 2013. Campact \& Co. Wie Hybridorganisationen das Grassrootscampaigning verändern. In Grassroots-Campaigning, Hrsg. Rudolph Speth, 213-224. Wiesbaden: Springer VS.

Waters, Richard D., und Paul M. Jones. 2011. Using video to build an organization's identity and brand: a content analysis of nonprofit organizations' YouTube videos. Journal of Nonprofit \& Public Sector Marketing 23:248-268.

Wen, Zhuoyi, und Alice M.L. Chong. 2014. Legitimate deficit: competitive bidding in a residual and semidemocratic welfare society. VOLUNTAS: International Journal of Voluntary and Nonprofit Organizations 25:214-1234. 